\title{
Do Speakers Avoid Ambiguities During Dialogue?
}

\author{
Sarah L. Haywood, Martin J. Pickering, and Holly P. Branigan \\ University of Edinburgh, Edinburgh, United Kingdom
}

\begin{abstract}
What affects speakers' production of ambiguous utterances in dialogue? They might consider ease of production for themselves, or ease of comprehension for their addressees. Previous research has demonstrated that ease of production plays a role in speakers'syntactic choices, but that ease of comprehension does not. However, such studies have not employed dialogues that involve role swapping on a turn-by-turn basis. In our experiment, participants alternated in giving and following instructions to move objects around on a grid. They tended to repeat the syntactic form just used by their interlocutor, reflecting sensitivity to ease of production. More interestingly, they were more likely to disambiguate their utterances when the visual context was potentially ambiguous than when it was not, reflecting sensitivity to ease of comprehension. We conclude that speakers pay attention to their beliefs about their addressees' ease of comprehension, in addition to considering ease of production for themselves.
\end{abstract}

Natural language is often ambiguous. Sometimes the ambiguity is only temporary, so that the correct interpretation of the utterance eventually becomes clear. For example, when a listener hears Put the penguin in the cup..., the phrase in the cup might indicate either where to put the penguin (Put the penguin in the cup now) or where the penguin currently is (Put the penguin that's in the cup onto the star). A listener who makes the wrong decision about the speaker's intended sentence structure can find it difficult to recover (i.e., the listener is syntactically "garden-pathed"; Rayner, Carlson, \& Frazier, 1983). Such difficulty can be avoided if the speaker explicitly disambiguates the utterance, for example, by exploiting optional phrases like that's (Put the penguin that's in the cup onto the star). But

Address correspondence to Sarah L. Haywood, Department of Psychology, School of Philosophy, Psychology, and Language Sciences, University of Edinburgh, 7 George Square, Edinburgh, EH8 9JZ, United Kingdom; e-mail: sarah.haywood@ed.ac.uk. sometimes an ambiguous form is easier to produce than an unambiguous form (e.g., because the construction involved is easier to access), so speakers might choose ambiguous forms even if their utterances are then difficult to understand. In this article, we consider whether speakers' syntactic choices in dialogue reflect ease of production, ease of comprehension, or both.

Speakers have available to them a number of syntactic strategies for avoiding ambiguities, but current evidence suggests that they do not use them. Ferreira and Dell (2000) found that participants were no more likely to include an optional that (after the verb) in temporarily ambiguous utterances like The coach knew you missed practice than in unambiguous utterances like The coach knew I missed practice. (The ambiguity occurs because, as the sentence unfolds, you could be incorrectly interpreted as a direct object, as in The coach knew you very well.) Likewise, speakers do not avoid the temporary ambiguity of The judge sent the letter to the president to the members of the congressional subcommittee by changing it into The judge sent the members of the congressional subcommittee the letter to the president, which has the same meaning but is not temporarily ambiguous (Arnold, Wasow, Asudeh, \& Alrenga, 2004). Finally, Kraljic and Brennan (2005) found no evidence that speakers avoid producing syntactically ambiguous utterances like Put the dog in the basket on the star when the visual context allows the listener to interpret in the basket as information about either which dog is to be moved or where the dog should be put. Thus, previous research suggests that speakers do not pay much attention to ease of comprehension when it comes to choosing between ambiguous and unambiguous forms.

Speakers' syntactic choices do, however, seem to be influenced by ease of production. One factor known to reflect ease of syntactic production both in monologue (Bock, 1986) and in dialogue (Branigan, Pickering, \& Cleland, 2000) is syntactic priming: the tendency to repeat syntactic structure. Ferreira (2003) found that speakers can be primed (in monologue) to produce temporarily ambiguous sentences like The mechanic mentioned the car could use a tune-up (the car could be the object of mentioned or the subject of could) and unambiguous 
sentences involving that (The mechanic mentioned that the car could use a tune-up). If similar effects occur in dialogue, speakers might tend to produce an ambiguous sentence like Put the penguin in the cup on the star after hearing an ambiguous prime (Put the pig on the block on the heart); conversely, they might produce an unambiguous sentence like Put the penguin that's in the cup on the star after hearing an unambiguous prime (Put the pig that's on the block on the heart). In some circumstances, priming might lead speakers to produce sentences that are difficult to interpret; that is, ease of production may lead to difficulty in comprehension.

We report an experiment that investigated how factors relating to ease of production and comprehension affect speakers' production of ambiguities during dialogue. Our experiment focused on utterances whose syntactic structure was ambiguous but whose interpretation was disambiguated by visual context (i.e., what the listener could see) and task constraints. Participants played a communication game that involved moving objects around a grid. Unlike previous experiments investigating avoidance of ambiguity, this study employed a game involving role swapping on a turn-by-turn basis: Participants alternated between giving instructions to their partner and carrying out their partner's instructions. Such dynamic shifting of roles (in particular, acting as the instruction follower on every other turn) may help raise speakers' awareness of the need to be unambiguous in their instructions.

One player was a confederate who was scripted to produce utterances, some of which were syntactically ambiguous (e.g., Put the pig on the block on the heart, when on the block could indicate either which pig was to be moved or where a pig should be put). The confederate explicitly disambiguated the syntax in half of his utterances, using that's (e.g., Put the pig that's on the block on the heart). On the following turn, participants had to give an instruction specifying an object and the position to which it should be moved. On turns with an unambiguous (onereferent) context, the array supported only one interpretation of the utterance (e.g., Put the penguin in the cup on the star, when the array contained one penguin and one cup, and the penguin was standing in the cup); on turns with an ambiguous (tworeferent) context, the array supported two interpretations (e.g., the array contained a penguin standing in a cup, a second penguin standing alone, and an empty cup). For half of the participants, the confederate was helpful, including that's only in ambiguous (two-referent) contexts; for the other half of the participants, the confederate was unhelpful, including that's only in ambiguous (one-referent) contexts.

Utterances were analyzed for evidence of priming (using that's more often after hearing a prime containing that's) and an effect of context (using that's more often in two-referent contexts, in which the addressee could be confused by an ambiguous construction, than in one-referent contexts); note that the ambiguity was always quickly resolved by the referential context (see Tanenhaus, Spivey-Knowlton, Eberhard, \& Sedivy,
1995). If speakers try to formulate utterances that are easy for addressees to understand, then participants would be expected to use that's in their instructions more often in two-referent contexts than in one-referent contexts. But if production favors ease of processing (for the speaker) over ease of comprehension, then one might expect a priming effect but no effect of referential context.

\section{METHOD}

\section{Participants}

Thirty-two native English-speaking students from the University of Edinburgh, Scotland, were paid to participate.

\section{Stimuli}

Two sets of instruction cards were created, prime cards and target cards. Sentences for the confederate to produce aloud were printed on the prime cards. There were 24 experimental primes, all of the form "Put the [name of object] (that's) in/on the [name of container] on the [name of shape]." Half of these primes contained that's, and half did not. Twenty-four filler primes were also created. Half of the fillers included a container object (e.g., "Put the sheep on the plate on the circle"), and half did not ("Put the paper clip on the square"). The confederate's filler primes referred to different objects and containers than the experimental primes did, and never included that's.

Participants' target cards showed a miniature version of the grid (see Fig. 1), with color photographs in the various cells to represent each object's current position on the grid. On each card, one cell on the grid was highlighted with a yellow box to show which object the participant should tell his or her partner to move. One of the geometric shapes was also highlighted, to show the object's target location. For example, yellow boxes around a penguin in a cup and around a star would indicate that the participant should tell his or her partner to Put the penguin (that's) in the cup on the star. Primes and targets were printed on paper, one instruction per page, and filed in separate ring binders for the confederate and participant.

Forty-eight items (24 experimental pairs and 24 fillers) were created by pairing a prime card from the confederate's set with a target card from the participant's set. The objects mentioned in a prime card and the to-be-described objects on the paired target card were always different. The items were assigned to $12 \mathrm{ex}-$ perimental rounds (2 experimental items and 2 fillers per round), which were presented in a different random order for each participant.

\section{Apparatus and Procedure}

The game was played on a $5 \times 5$ grid; eight of the cells contained geometric shapes. The confederate and the participant sat facing the grid, with a vertical screen between them so that neither could see the other person or that person's instruction 


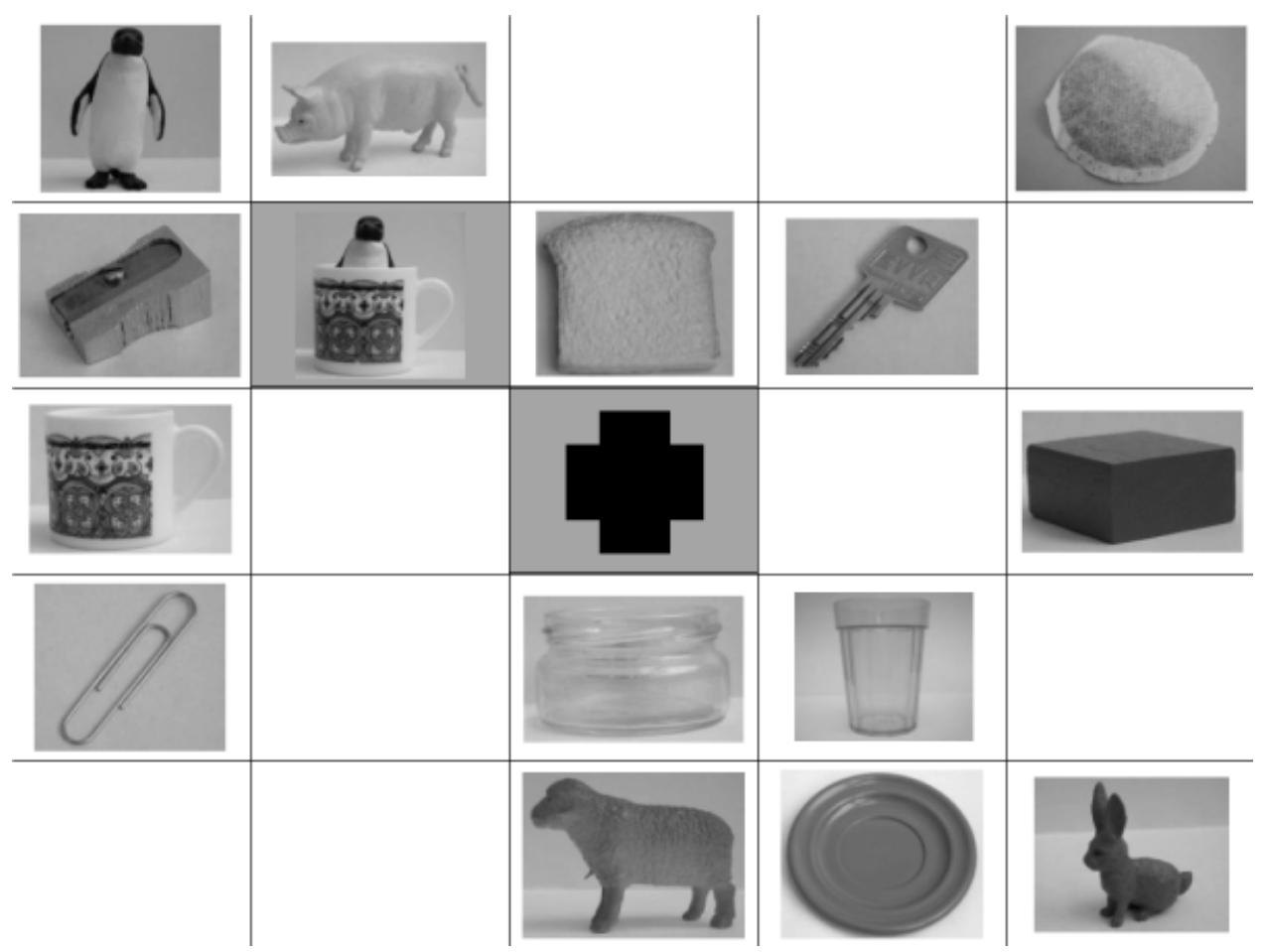

Fig. 1. Sample target card. (Color photographs were used in the experiment.) This card comes from the end of a round, when there was only one geometric shape left to be covered up with an object.

cards (see Fig. 2). The experimenter explained that the task was to help the partner move objects around on the grid as quickly and as accurately as possible; if either player did not understand an instruction, he or she could say, "Please repeat." The experimenter explained that the to-be-moved object would sometimes be inside or on top of something else, but that only the topmost object should be moved; the container object itself was to stay behind. The experimenter changed the arrays between rounds; objects not in use on a particular round were kept out of sight in the experimenter's box.

The confederate gave the first instruction in each round. The same person played the confederate throughout the experiment and was trained to use a flat intonation for all instructions. The confederate behaved throughout as a naive participant; postexperimental questionnaires showed that none of the participants was aware that he was a confederate. Participants' responses were recorded on audiotape.

\section{Coding and Analysis}

We coded participants' target responses as "with that's" if they were of the form "Put the [name of object] that's in/on the [name of container] on the [name of shape]." Target responses with the same form but without that's were coded as "without that's." All other responses were coded as "other." "Other" responses comprised target responses not preceded by the intended prime (usually because the participant turned over two pages in the instruction folder by mistake), target responses in which the participant did not mention the container object (e.g., Put the penguin on the star), and target responses that included disambiguating words other than that's (e.g., from, which is, standing in, off). "Other" responses ( $10 \%$ of the data) were excluded from our analyses, so that the proportions of "with that's" and "without that's" responses are complementary. Participant $\left(F_{1}\right)$ and item $\left(F_{2}\right)$ means for each experimental condition were submitted to separate analyses of variance (ANOVAs), with prime (without that's vs. with that's) and context (one-referent vs. two-referent context) as within-participants and within-items factors. Helpfulness (helpful vs. unhelpful confederate) was included as a between-participants and within-items factor.

\section{RESULTS}

Overall, participants included that's in $32 \%$ of their utterances. They produced more that's following primes containing that's (46\%) than following primes without that's (18\%; see top half of Table 1$)$. This $28 \%$ effect of prime was significant, $F_{1}(1,30)=$ $31.65, p<.001, \eta^{2}=.513 ; F_{2}(1,23)=26.74, p<.001, \eta^{2}=$ .538. Participants also showed a sensitivity to referential context. They produced more that's in ambiguous two-referent contexts $(36 \%)$ than in unambiguous one-referent contexts $(28 \%)$. This $8 \%$ effect of context was also significant, $F_{1}(1,30)$ $=7.46, p=.01, \eta^{2}=.199 ; F_{2}(1,23)=5.91, p<.05, \eta^{2}=$ 


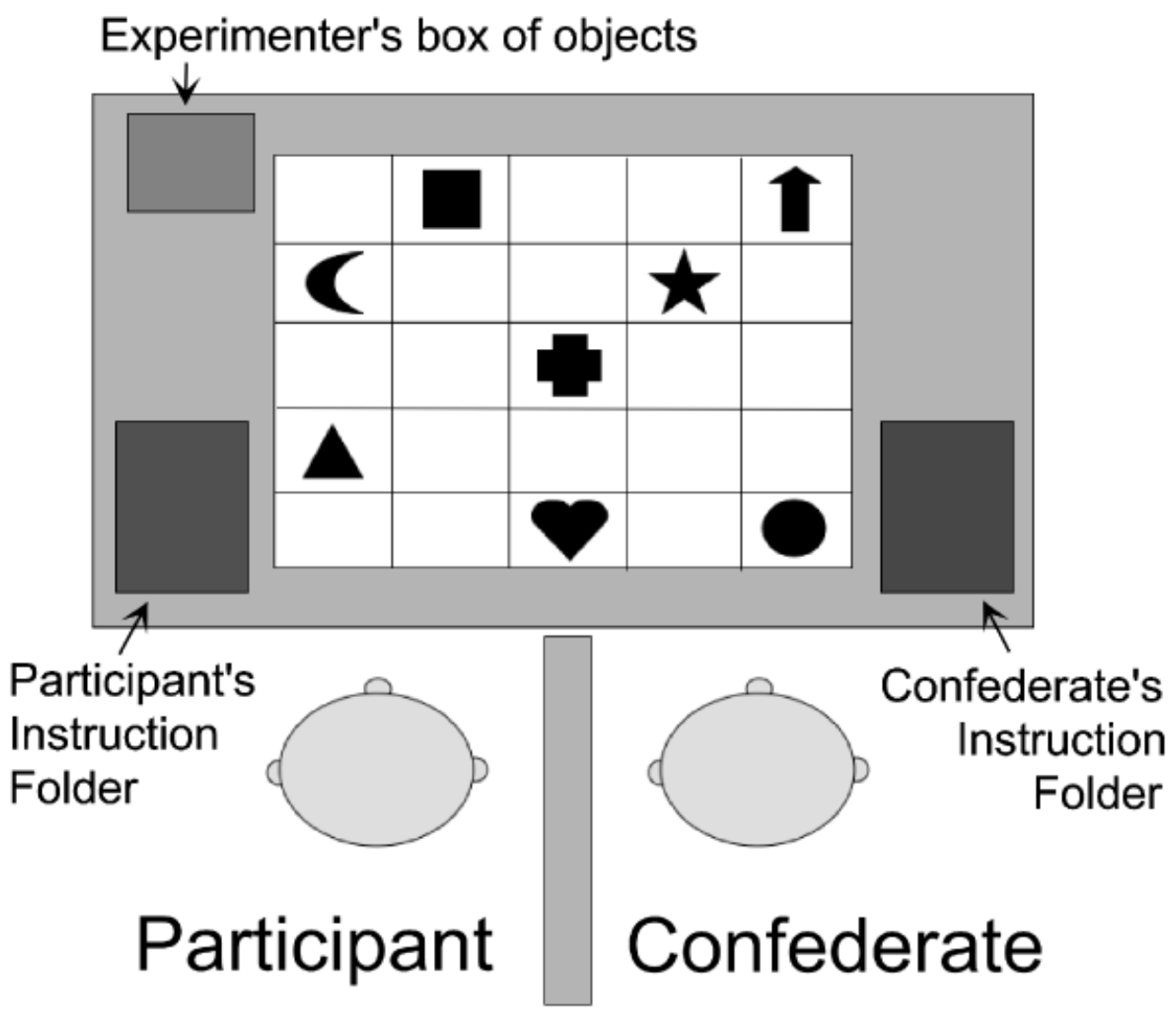

Fig. 2. Overhead view of the experimental setup.

.204. There was no interaction between prime and context (both $\left.F_{\mathrm{s}}<1\right)$; that is, these effects were independent.

Participants in the helpful-confederate condition produced more that's $(36 \%)$ than participants in the unhelpful-confederate condition (29\%); this effect was significant by items but not

TABLE 1

Proportion of Target Responses Including That's or Any Disambiguating Word

\begin{tabular}{lcc}
\hline \hline Condition & $\begin{array}{c}\text { Helpful } \\
\text { confederate }\end{array}$ & $\begin{array}{c}\text { Unhelpful } \\
\text { confederate }\end{array}$ \\
\hline \multicolumn{1}{c}{ Responses with that's } & \\
Prime without that's & $.13(.20)$ & $.17(.26)$ \\
$\quad \begin{array}{l}\text { Unambiguous (one-referent) context } \\
\text { Ambiguous (two-referent) context }\end{array}$ & $.25(.30)$ & $.17(.26)$ \\
$\begin{array}{l}\text { Prime with that's } \\
\text { Unambiguous (one-referent) context }\end{array}$ & $.50(.38)$ & $.33(.32)$ \\
Ambiguous (two-referent) context & $.53(.40)$ & $.49(.38)$ \\
\hline
\end{tabular}

Responses with any disambiguating word or words

Prime without that's

$\begin{array}{lll}\text { Unambiguous (one-referent) context } & .15(.19) & .17(.26) \\ \text { Ambiguous (two-referent) context } & .29(.30) & .18(.26) \\ \text { Prime with that's } & & \\ \text { Unambiguous (one-referent) context } & .50(.38) & .33(.32) \\ \text { Ambiguous (two-referent) context } & .60(.38) & .50(.38)\end{array}$

Note. Standard deviations are in parentheses. by participants, $F_{1}(1,30)=0.490, p=.50, \eta^{2}=.016 ; F_{2}(1$, $23)=8.64, p<.01, \eta^{2}=.273$. There was no interaction between prime and helpfulness, $F_{1}<1 ; F_{2}(1,23)=3.01, p=.10$, $\eta^{2}=.116$, nor, crucially, between helpfulness and context (both $F_{\mathrm{s}}<1$ ); that is, the confederate's helpfulness did not affect the participant's tendency to disambiguate. The threeway interaction was significant, $F_{1}(1,30)=4.69, p<.05, \eta^{2}=$ $.135 ; F_{2}(1,23)=4.32, p<.05, \eta^{2}=.158 .^{1}$

The lower half of Table 1 shows the proportion of target responses that were disambiguated by any optional words (e.g., "Put the pig from the block on the star," "Put the pig which is standing on the block on the star," "Put the pig that's on the block on the star"). An analysis based on these data found an identical priming effect (28\% more disambiguated utterances following primes containing that's than following primes not containing that's) and a marginally stronger effect of referential context $(10 \%$ more disambiguated utterances in two-referent contexts than in one-referent contexts). The pattern of main effects and interactions was the same as in the previous set of

\footnotetext{
${ }^{1}$ A second experiment in which each item appeared in only one condition (hence, prime and context were between-items factors) yielded a $27 \%$ priming effect (significant by participants and items) and a 7\% context effect (significant by participants and marginal by items, $p=.08$ ). There was also a $7 \%$ effect of helpfulness (significant by items alone). There was no interaction between helpfulness and context (both $F_{\mathrm{s}}<1$ ), nor a three-way interaction (both $F_{\mathrm{s}}<1$ ), suggesting that the (unpredicted) three-way interaction in the main experiment was a Type I error.
} 
analyses, except that the three-way interaction was marginal, $F_{1}(1,30)=3.79, p=.06, \eta^{2}=.112 ; F_{2}(1,23)=3.60, p=.07$, $\eta^{2}=.135$.

Postexperimental questionnaires showed that participants were unaware of the syntactic ambiguity in the utterances and unaware of the manipulation of the confederate's helpfulness (i.e., they did not comment on their partner's consistent use or lack of use of that's).

\section{DISCUSSION}

Our data show clear evidence for two independent influences on ambiguity avoidance in dialogue: syntactic priming and referential context. Hence, speakers' choices reflected both ease of production and ease of comprehension.

Participants were primed by their interlocutor to produce ambiguous or disambiguated syntactic forms. Our results add to the growing body of evidence that priming is a pervasive influence in dialogue (see Pickering \& Garrod, 2004); specifically, they demonstrate that syntactic priming influences the production of potentially ambiguous sentences in dialogue (cf. Ferreira, 2003). But even in the context of a strong priming effect, participants also showed a sensitivity to referential context in their avoidance of syntactic ambiguity. They were more likely to produce disambiguated utterances when the addressee's visual array could potentially lead to confusion than when it could not. Numerically, the priming effect was substantially larger than the context effect, suggesting that factors relating to ease of production may have a stronger influence on language production than factors relating to ease of comprehension. Crucially, however, we have demonstrated that the two kinds of factors operate independently in the same task.

Our data show that language production is influenced by visual context. The influence of visual context on comprehension has been explored extensively and is well established (see, e.g., Chambers, Tanenhaus, Eberhard, Filip, \& Carlson, 2002), but the influence of visual context has been less explored in production. By itself, the sentence fragment Put the penguin in the cup. . . is syntactically ambiguous whether the addressee's array of objects contains one or two penguins. Yet our participants responded differently to these two kinds of visual context, perhaps because the presence of more than one potential referent in the two-referent case cued them to the need to be explicit in their instruction. We interpret our results as evidence that speakers can be sensitive to potential ambiguities, and that they try explicitly to disambiguate their utterances when the visual context affords the opportunity for miscommunication.

Previous research has failed to find effects of linguistic or referential context on the production of optional disambiguating words (Ferreira \& Dell, 2000; Kraljic \& Brennan, 2005). Such failures may reflect differences in experimental tasks. Speakers may pay relatively little attention to ease of comprehension when they have no visual cues to the linguistic ambiguity, when there are no obvious consequences of being ambiguous, or when they do not exchange roles with another person turn-by-turn. In contrast, in a role-swapping task like ours, considerations of the addressee may be particularly salient. Hence, we suggest that the tension between what is easy for the speaker and what is "best" for the addressee may not always be resolved the same way; instead, speakers may adjust their behavior in response to the communicative situation.

Acknowledgments - We thank Bryn Harris, Nivja de Jong, Susan Brennan, and an anonymous reviewer. S.L.H. acknowledges a Postgraduate Research Studentship and a Postdoctoral Research Fellowship from the Economic and Social Research Council.

\section{REFERENCES}

Arnold, J.E., Wasow, T., Asudeh, A., \& Alrenga, P. (2004). Avoiding attachment ambiguities: The role of constituent ordering. Journal of Memory and Language, 51, 55-70.

Bock, J.K. (1986). Syntactic persistence in language production. Cognitive Psychology, 18, 355-387.

Branigan, H.P., Pickering, M.J., \& Cleland, A.A. (2000). Syntactic coordination in dialogue. Cognition, 75, B13-B25.

Chambers, C.G., Tanenhaus, M.K., Eberhard, K., Filip, H., \& Carlson, G. (2002). Circumscribing referential domains in real time language comprehension. Journal of Memory and Language, 47, 30-49.

Ferreira, V.S. (2003). The persistence of optional complementizer production: Why saying "that" is not saying "that" at all. Journal of Memory and Language, 48, 379-398.

Ferreira, V.S., \& Dell, G.S. (2000). Effect of ambiguity and lexical availability on syntactic and lexical production. Cognitive Psychology, 40, 296-340.

Kraljic, T., \& Brennan, S.E. (2005). Prosodic disambiguation of syntactic structure: For the speaker or for the addressee? Cognitive Psychology, 50, 194-231.

Pickering, M.J., \& Garrod, S. (2004). Toward a mechanistic psychology of dialogue. Behavioral and Brain Sciences, 27, 169-225.

Rayner, K., Carlson, M., \& Frazier, L. (1983). The interaction of syntax and semantics during sentence processing. Journal of Verbal Learning and Verbal Behavior, 22, 358-374.

Tanenhaus, M.K., Spivey-Knowlton, M.J., Eberhard, K.M., \& Sedivy, J.C. (1995). Integration of visual and linguistic information in spoken language comprehension. Science, 268, 1632-1634.

(RECEIVED 5/20/04; REVISION ACCEPTED 7/26/04) 